\title{
IN VIVO TRACKING OF SEGMENTAL BONE DEFECT HEALING REVEALS THAT CALLUS PATTERNING IS RELATED TO EARLY MECHANICAL STIMULI
}

\author{
Manav Mehta ${ }^{1,3 *}$, Sara Checa ${ }^{1}$, Jasmin Lienau ${ }^{1}$, Dietmar Hutmacher ${ }^{4}$ and Georg N. Duda ${ }^{1,2}$ \\ ${ }^{1}$ Julius Wolff Institute and Centre for Musculoskeletal Surgery, Charité - Universitätsmedizin Berlin, Germany \\ ${ }^{2}$ Berlin-Brandenburg Centre for Regenerative Therapies, Charité - Universitätsmedizin Berlin, Germany \\ ${ }^{3}$ School of Engineering and Applied Sciences, Harvard University, Cambridge, MA, USA \\ ${ }^{4}$ Queensland University of Technology, Brisbane, Australia
}

\begin{abstract}
This study addresses the hypothesis that callus formation, patterning, and mineralisation are impaired during the early phase of critical sized bone defect healing, and may relate to inter-fragmentary tissue strains within the bone defect area. Twenty four 12 week old Sprague Dawley rats were used for this study. They were divided into two groups defined by the femur bone defect size: (i) $1 \mathrm{~mm}$ resulting in normal healing $(\mathrm{NH})$, and (ii) a large sized $5 \mathrm{~mm}$ defect resulting in critical healing $(\mathrm{CH})$. Callus formation, patterning, and mineralisation kinetics in both groups were examined in the periosteal and osteotomy gap regions using a novel longitudinal study setup. Finite element analyses on $\mu \mathrm{CT}$ generated tomograms were used to determine inter-fragmentary tissue strain patterns and compared to callus formation and patterning over the course of time. Using a novel longitudinal study technique with $\mu \mathrm{CT}$, in vivo tracking and computer simulation approaches, this study demonstrates that: (i) periosteal bone formation and patterning are significantly influenced by bone defect size as early as 2 weeks; (ii) osteotomy gap callus formation and patterning are influenced by bone defect size, and adapt towards a non-union in critical cases by deviating into a medullary formation route as early as 2 weeks after osteotomy; (iii) the new bone formation in the osteotomy gap enclosing the medullary cavity in the $\mathrm{CH}$ group is highly mineralised; (iv) inter-fragmentary strain patterns predicted during the very early soft callus tissue phase (less than 2 weeks) are concurrent with callus formation and patterning at later stages. In conclusion, bone defect size influences early onset of critical healing patterns.
\end{abstract}

Key words: In vivo imaging; bone healing; non-unions; segmental bone defect; micro-computed tomography; finite element; critical healing patterns.

*Address for correspondence:
Dr Manav Mehta
Julius Wolff Institute and Centre for Musculoskeletal
Surgery
Charité - Universitätsmedizin Berlin
Augustenburger Platz 1,
D-13353 Berlin
Germany

Telephone Number: +49 (0)30-450 559048

FAX Number: +49 (0)30-450 559969

E-mail: manav.mehta@charite.de

Website: jwi.charite.de

\section{Introduction}

Post-traumatic fracture non-unions have been clinically defined as a lack of radiographic bony bridging, failure to reach a fracture stiffness of $7 \mathrm{Nm}$ per degree even after week 20, and failure of functional weight bearing by 6 months (Marsh, 1998). Although such clinical diagnoses are available on fracture non-unions, an indepth quantitative characterisation of their pathological onset and aetiology remains absent. In a recent study to establish a standardised atrophic non-union rat model using a critical sized bone defect (Mehta et al., 2011b), clear differences in healing patterns in the periosteal and osteotomy gap callus regions between normal $(1 \mathrm{~mm}$ bone defect size) and critical healing ( $5 \mathrm{~mm}$ large bone defect size) groups were qualitatively highlighted. Whilst $2 \mathrm{D}$ radiographs in the normal healing group showed a periosteal and endosteal callus bridging, the critical healing group indicated a cessation of periosteal and endosteal healing without any progressive response towards bony bridging. Furthermore, histological analysis of the fracture callus samples from critical healing subjects revealed highly organized lamellar bone sealing the medullary cavity. Additionally, the non-mineralised callus within the osteotomy gap mainly consisted of scar tissue (fibrous tissue) and lacked any signs of cartilage formation and endochondral ossification. Such qualitative experimental evidence on healing patterns during non-unions in bone defect healing led us to formulate the following questions (Fig. 1): What is the difference between a normal and a large bone defect with respect to formation rate and patterning, specifically (i) of the periosteal callus and (ii) in the osteotomy gap? (iii) What is the extent of mineralisation of the bony outgrowth enclosing the medullary cavity, and (iv) what is the influence of interfragmentary strains on the non-healing situation?

It is widely known that an optimal local mechanical environment, which is determined by fixation stability, is crucial for successful healing (Claes et al., 1998; Duda et al., 1998; Kenwright and Goodship, 1989; Lacroix and Prendergast, 2002; Mehta et al., 2011a; Mehta et al., 2010). Initial instability can delay bone healing or lead to non-unions, due to rupturing of the bridging callus tissue and of the nutrient blood vessels (Kraus et al., 1999; Mehta et al., 2010). In contrast, if mechanical stimuli were to be completely absent, a delayed bone healing would be observed (Goodship and Kenwright, 1985; Kraus et al., 1999). Several studies investigated the effect of bone defect size on mechanical conditions within the fracture gap region. For example, Claes et al. quantified the inter- 


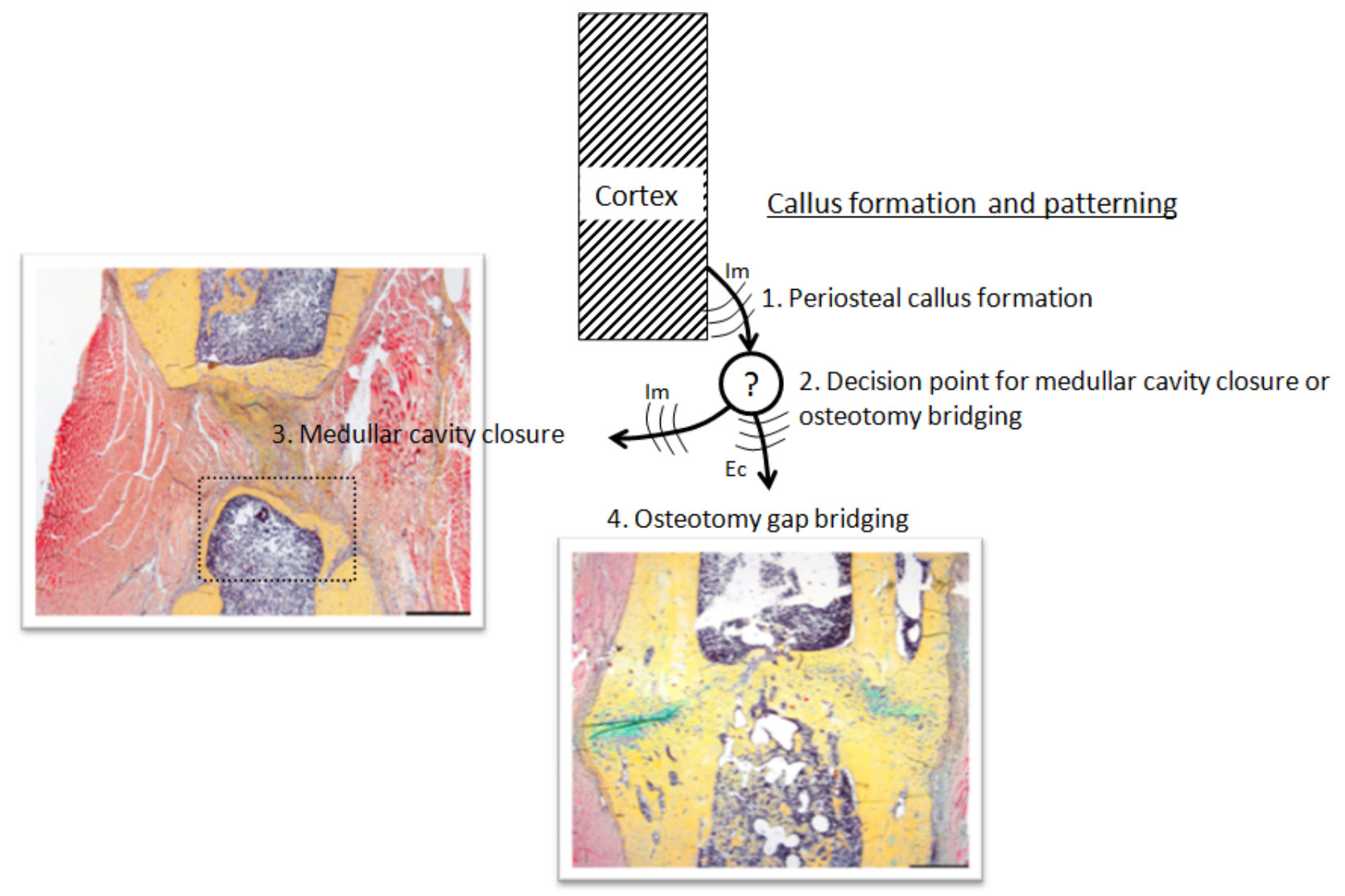

Fig. 1. Impaired callus formation and patterning during the development of non-unions. Movat Pentachrome stained frontal sections through the osteotomy region 6 weeks after surgery demonstrating differences in periosteal and osteotomy gap callus healing patterns between normal ( $1 \mathrm{~mm}$ bone defect size) and critical healing ( $5 \mathrm{~mm}$ large bone defect size). However, an understanding of critical healing patterns, its pathological onset and aetiology remained absent due to lack of in-depth spatiotemporal characterisations during the development of a non-union. Im: intramembranous bone formation, Ec: endochondral bone formation. Histological images modified from (Mehta et al., 2011b).

fragmentary movement for different fixator stiffness and gap sizes (Claes et al., 1997). However, to our knowledge the strain distributions in a large sized bone defect model has not been reported previously, neither has it been correlated to patterns of newly formed bone during the course of healing using experimental data (Reichert et al., 2012).

3D micro computed tomography $(\mu \mathrm{CT})$ characterisation allows in-depth analyses of fracture bony callus structure and composition providing good assessment of callus mechanical competence during fracture healing. In vitro $\mu \mathrm{CT}$ studies have shown mechanical competence of bone tissue to be highly dependent on the size, geometry and micro-structure of mineralised tissue as well as its mineral density (Mehta et al., 2011a; Mehta et al., 2010; Morgan et al., 2009). Heterogeneity of mineralisation (tissue mineral density distribution, TMDD), together with its spatial distribution, have been used to describe bone material quality and associated with mechanical properties of bone tissue (Roschger et al., 1998; Ruffoni et al., 2007). So far, these parameters have been only characterised at endpoint post mortem, and are missing a spatial-temporal characterisation of callus tissue as it develops over time.

Newer in vivo $\mu \mathrm{CT}$ scanning technologies allow for spatiotemporal monitoring of individual animals over time.
With the disadvantages of (i) lower resolution images, (ii) lower signal to noise ratio, and (iii) ionising radiations, in vivo monitoring studies overcome the disadvantages that are commonly associated with endpoint in vitro characterisations. Longitudinal $\mu \mathrm{CT}$ studies (4D) in bone healing have enabled early and more frequent evaluation and monitoring of mineralised callus tissue formation with a possibility of region specificity (van Lenthe and Muller, 2008). However, region specific analyses rely on localisation of a volume of interest (VOI) during each scan in longitudinal monitoring and the use of post image registration techniques. To achieve region-specific analysis (periosteal and osteotomy gap callus characteristics in 3D), and to identify the onset of critical healing patterns due to large bone defect healing, an in vivo $\mu \mathrm{CT}$ setup with adjusted localisation of VOI has been used in the present study to longitudinally monitor femoral bone defects in rats.

Our study hypothesis was that periosteal- and osteotomygap callus formation, patterning and mineralisation would be impaired from the early phase of critical sized bone defect healing onwards, and that mineralised callus tissue patterning would be related to inter-fragmentary tissue strain patterns resulting within the bone defect area. To test our hypothesis, the study aim was to develop hardware and 

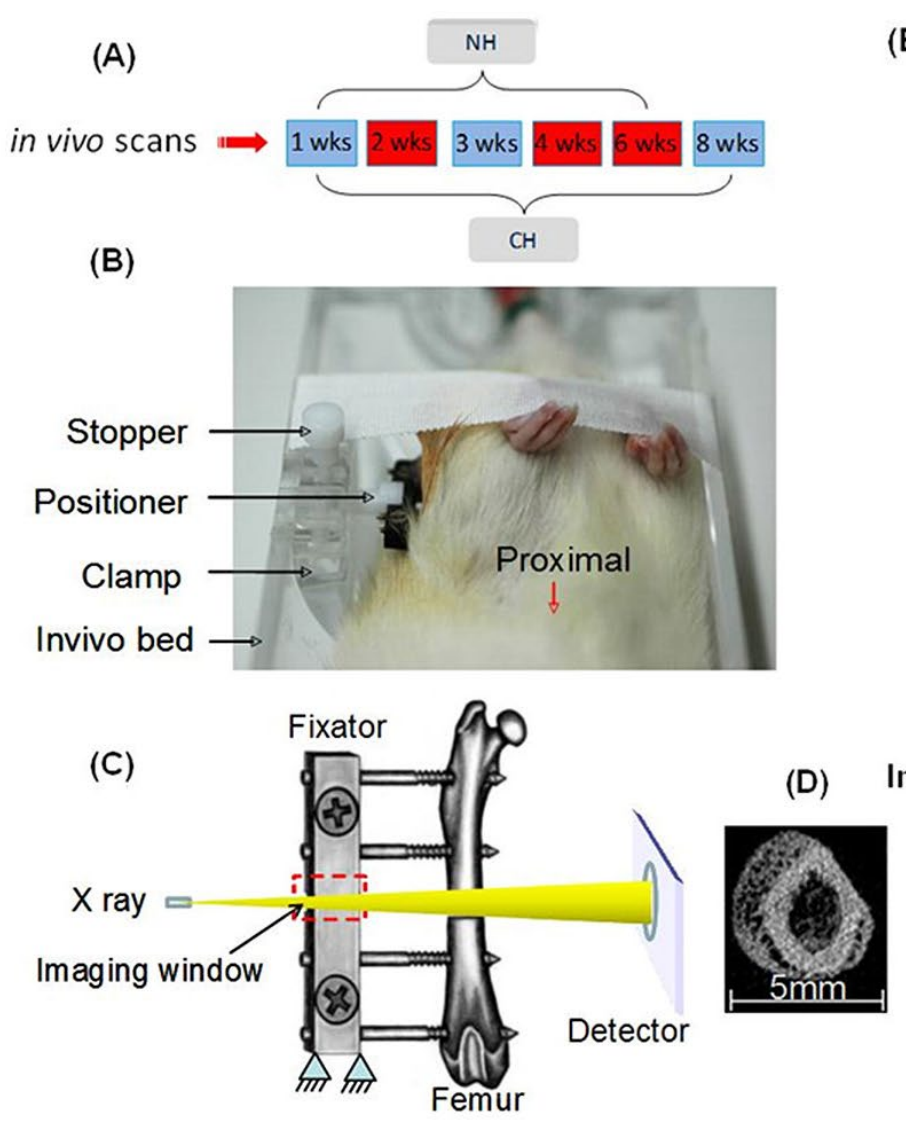

(E)

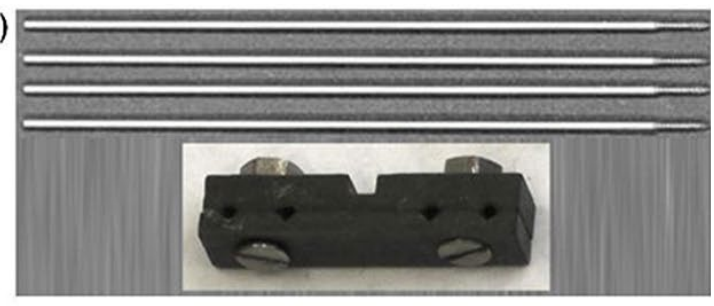

(F)

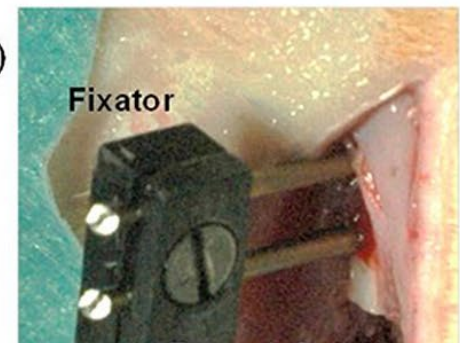

maging window

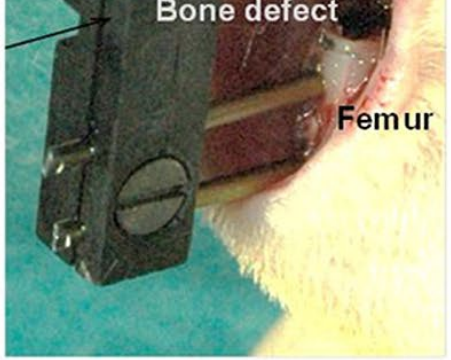

Fig. 2. In vivo imaging setup for scanning femoral bone defects with a repeatable VOI. Image (A) illustrates the scanning intervals for the two groups. Red indicates in vivo monitoring scans at 2, 4 and 6 weeks post osteotomy. Blue indicates more frequent scanning at 1, 2, 3, 4, 6, and 8 weeks post operatively. Image (B) shows an anaesthetised rat placed into an imaging-bed with its external femoral fixator positioned into a clamp and stopper setup. The clamp, stopper and positioner allow the fixator to be plugged, rotated, aligned and tightly fastened such that the scanning VOI and the scanner axis are aligned. The clamp, stopper and positioner provide accurate and repeatable placement of the scanning VOI during monitoring of individuals over time. Image (C) shows a schematic illustration of an external fixator with an imaging window designed between the fixator pins that allows for minimally attenuated passage of X-rays through the carbon material into the volume of interest, and providing images free of artefacts (image D) at the detector. Image (E) shows components of the custom-made external fixator - top image shows titanium K-wires to fix the fixator bar to the bone; bottom image shows the carbon fixator with its 2-screws and nuts used to clamp and secure the entire fixator assembly of cross bars and stainless steel pins to the bone. Image (F) is a photograph showing the external carbon fixator in situ attached to the femur of a rat from the $\mathrm{CH}$ group.

software methods to enable in vivo tracking of bone defect healing and quantitatively compare 3D callus formation, patterning, and mineralisation kinetics in a region specific manner (periosteal and osteotomy gap regions) between a normal (1 mm bone defect) and a critical bone healing model ( $5 \mathrm{~mm}$ bone defect). Finite element analysis was used to investigate the relation between inter-fragmentary tissue strain distributions and callus tissue formation over the course of time, with a focus on the early healing period.

\section{Materials and Methods}

\section{Animals and experimental design}

All animal experiments were carried out according to the policies established by the Animal Welfare Act, the NIH Guide for Care and Use of Laboratory Animals and the National Animal Welfare Guidelines, and were approved by the local legal representative (Landesamt für Gesundheit und Soziales, Berlin, Reg.no.: G0071/07).

Twenty four 12 week-old female Sprague Dawley rats were used for this study. They were divided into two groups with twelve rats in each. The groups were defined by the femoral bone defect size: (i) $1 \mathrm{~mm}$ - normal healing (NH), and (ii) a large sized $5 \mathrm{~mm}$ - critical healing $(\mathrm{CH})$. Based on a previous study (Mehta et al., 2011b), the time from surgery to sacrifice was set at 6 weeks without restriction in weight bearing in the $\mathrm{NH}$ group, and for an additional 2 weeks in $\mathrm{CH}$ group to monitor and verify the development of a non-union. All subjects underwent longitudinal in vivo monitoring scanning at 2, 4 and 6 weeks post osteotomy (Fig. 2A). Five rats out of each group received more frequent scanning at 1, 2, 3, 4, 6 and 8 weeks post operatively (Fig. 2A). 
Fig. 3. Tissue mineralisation density distribution (TMDD) histogram, expressing the probability that a given bone voxel has a certain greyscale value depending on its attenuation.

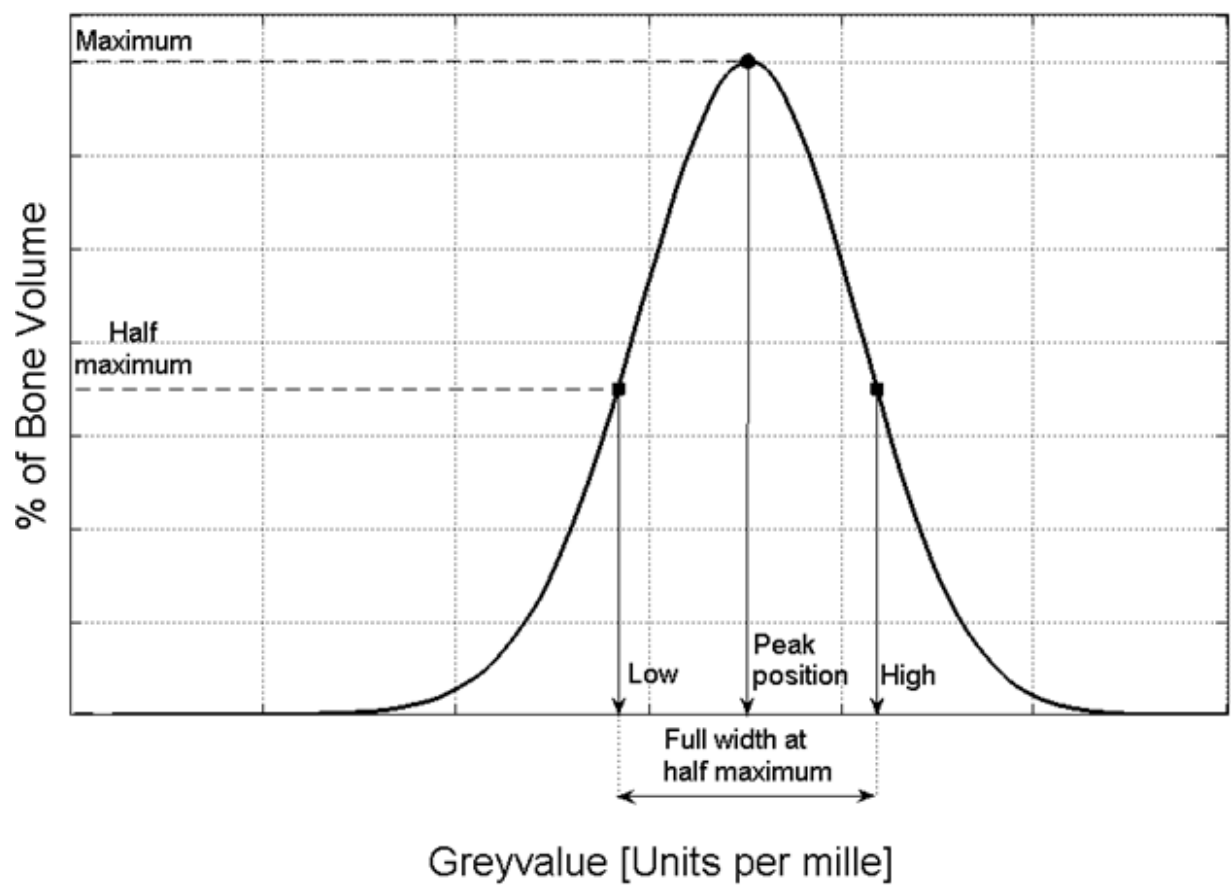

\section{External fixators and surgical procedure}

The custom-made external fixation device consisted of four titanium $1.2 \mathrm{~mm}$ diameter Kirschner (K)-wires (Medizintechnik Jagel, Bad Blankenbug, Germany) with a $5 \mathrm{~mm}$ thread and a crossbar composed of two carbon plates bound by two countersunk screws. The distance between fixator and bone (offset) was set to $7.5 \mathrm{~mm}$ (Figs. 2E and F).

The animal models for this study have been established and described in a previous study (Mehta et al., 2011b). Using a lateral approach, the left femur was osteotomised at the mid shaft and externally fixated, according to the method described earlier (Strube et al., 2008). Briefly, the external fixator was affixed to the lateral aspect of the femur using the fixator bar as a drill guide for the $\mathrm{K}$-wires. Animals in the $\mathrm{NH}$ group received a single transverse osteotomy between the two inner K-wires with a gap size of $1 \mathrm{~mm}$, while animals from group $\mathrm{CH}$ received a double transverse osteotomy with a gap size of $5 \mathrm{~mm}$. The fixator bar was mounted at a distance of $7.5 \mathrm{~mm}$ to the lateral surface of the bone (Fig. 2F), and the wound was then sutured in two layers. The animals were sacrificed at 6 weeks $(\mathrm{NH})$ or 8 weeks $(\mathrm{CH})$ post-surgery, and both femurs were harvested for further processing.

\section{Clinical evaluation}

Preoperatively, as well as weekly postoperatively, rectal temperature and body weight were measured to detect possible infections (temperature $\geq 38{ }^{\circ} \mathrm{C}$; weight loss $>10 \%$ of body weight over 1 week). Postoperatively, the animals were observed daily and if necessary analgesia was given. In case of complications, animals were sacrificed and excluded from the study.

\section{In vivo monitoring setup}

A radiolucent in vivo animal imaging-bed, made of acrylic, was developed to reproducibly position the subjects (Fig. 2B). A detachable modular clamp integrated into the imaging-bed helped position the fixator into the scanning volume of interest in a reproducible manner without imaging artefacts. The modular clamp enabled the scan axis to coincide nominally with the diaphyseal axis of femora. CT dosage index was calculated according to the specifications from the manufacturer to be $86 \mathrm{mGy}$ for a $35.5 \mu \mathrm{m}$ resolution scan, with integration time of $150 \mathrm{~ms}$, at a voltage of $55 \mathrm{kV}$, current of $145 \mu \mathrm{A}$ that lasted for $8.3 \mathrm{~min}$. One subject served as a reproducibility control for VOI localisation. The subject was removed from its scanning setup after each scan and placed again for 4 similar scans each time post sacrifice. At 6 weeks postoperative, callus volume fraction and tissue mineral density due to radiation between animals that received higher radiation from weekly scanning to those that received lower radiation from bi-weekly scanning were compared.

Descriptive histology was performed on callus sections from the NH group to screen for radiation-induced morphological tissue changes. Femora were dehydrated with alcohol and xylol, embedded in methylmethacrylate (Technovit 9100 NEU, Heraeus Kulzer GmbH, Hanau, Germany), cut into $6 \mu \mathrm{m}$-thick sections and stained with Movat Pentachrome.

Animals from the NH group were used to investigate information loss due to medium spatial resolution (35.5 $\mu \mathrm{m})$ during in vivo scanning on bone volume (BV) and tissue mineral density (TMD) parameters compared to high resolution $(10.5 \mu \mathrm{m})$ in vitro scanning. To achieve this, animals from the $\mathrm{NH}$ group were sacrificed after the last in vivo follow up scan at 6 weeks, the osteotomised and contralateral femur harvested and prepared for a highresolution in vitro scan. The scans were performed on a Viva40 micro-CT (Scanco Medical AG ${ }^{\circledR}$, Brüttisellen, Switzerland), at a voltage $70 \mathrm{KV}$ and current of $114 \mu \mathrm{A}$. Voxel size was selected to be isotropic and fixed at $10.5 \mu \mathrm{m}$. A threshold of $45 \%$ attenuation of intact bone was used for analysis of $\mathrm{BV}$ and TMD parameters. 
Mineral tissue distribution and kinetics were measured using volumetric bone tissue mineralisation density distribution (TMDD). The TMDD is a histogram expressing the probability that a given bone voxel has a certain greyscale value depending on its attenuation (Roschger et al., 1998; Ruffoni et al., 2007) (Fig. 3). These histograms were obtained from the segmented bone volume of mineralised callus tissue as it develops in vivo in an individual over the follow-up period. Using these in vivo histograms, it is possible to quantify (i) $\mathrm{TMDD}_{\text {peak }}$ : the peak height of the distribution (MaxFreq), having the dimension of $\%$ bone volume, and (ii) $\mathrm{TMDD}_{\text {f.h.width }}$ : the full width at half maximum of MaxFreq. The values have been reported as a percentage of intact bone.

\section{$\mu \mathrm{CT}$ analysis}

For callus patterning analysis, the post processing of $\mu \mathrm{CT}$ image stacks was performed using Amira ${ }^{\circledR}$ software (Indeed - Visual Concepts GmbH, Berlin, Germany) for image registration with region specificity over time. An in-house MATLAB script was used to characterise callus patterning of the periosteal region over time and reported in a polar coordinate system. For characterisation of $3 \mathrm{D}$ callus competence over time (i.e., size, geometry, and mineralisation), newly formed mineralised callus tissue was analysed. The VOI contained the 1 or $5 \mathrm{~mm}$ osteotomy gap region and $1 \mathrm{~mm}$ in the proximal and distal directions. Using a manually drawn counter-clockwise contour, the cortical bone was excluded from the VOI. The resulting greyscale images were segmented using a low pass Gaussian filter. A fixed global threshold of $545 \mathrm{mg} \mathrm{HA} / \mathrm{cm}^{3}$ was selected as the best choice that allowed the rendering of mineralised callus for the in vivo groups. The threshold for callus mineralisation was approximately $45 \%$ attenuation of intact bone (Duvall et al., 2007). The standard for comparison was set by visually evaluating 10 random single tomographic slices from four samples per group to isolate the mineralised tissue and preserve its morphology while excluding unmineralised tissues.

Using the processed images, the following morphometric measurements were analysed: (i) Bone volume (BV normalised against gap size), (ii) average polar moment of inertia (\% of contralateral femur from in vitro scans), and (iii) average tissue mineral density (\% of intact bone). Region specific analysis for periosteal and osteotomy gap areas are reported for these parameters. All analyses were performed on the digitally extracted callus tissue using 3D distance techniques (Scanco ${ }^{\circledR}$ software, Scanco Medical $\mathrm{AG}^{\circledR}$, Brüttisellen, Switzerland) (Hildebrand and Ruegsegger, 1997).

\section{Strain pattern prediction using finite element analysis}

To investigate the relationship between the local mechanical conditions and early callus formation and patterning, the mechanical strains within the callus region during the early phase of healing in the uneventful (NH group, $1 \mathrm{~mm}$ ) and large defect healing model ( $\mathrm{CH}$ group, $5 \mathrm{~mm}$ ) were determined using finite element analysis. Voxel-based finite element models of the frontal mid-cross sectional plane were derived from the $\mu \mathrm{CT}$-images of exemplary individuals from each group at 1 and 2 week time points. Material properties of the cortex and newly formed callus were derived using CT-derived greyscale values. The soft tissue region (native value $<10176$ ) was assigned an elastic modulus of $0.5 \mathrm{MPa}$ (Leong and Morgan, 2008). Highly mineralised bone (native value $>16403$ ) was assigned $15 \mathrm{GPa}$. $5 \mathrm{GPa}$ was assigned to low mineralised bone (native value equal to 10176) and a linear function was used for intermediate values (Shefelbine et al., 2005).

To determine the inter-fragmentary movement (IFM) for the two gap sizes, an already developed threedimensional finite element model of the femoral osteotomy (Checa et al., 2011) was used. This model includes the bone, the callus and the external fixator, allowing for the determination of how the different gap sizes result in different IFMs under the same loading and fixation conditions. IFMs predicted in such a way were comparable to those measured experimentally in a large animal model (Checa et al., 2011). A compression load equivalent to 6-times animal body weight during normal walking was applied (Wehner et al., 2010). The calculated IFMs (0.05 and $0.035 \mathrm{~mm}$ in the uneventful and critical healing models, respectively) were thereafter applied as displacement boundary conditions to the $2 \mathrm{D} \mu \mathrm{CT}$-image-derived finite element models.

\section{Statistical analyses}

All collected data of the in vivo measurements are reported as median values with $25-75$ percentiles for each group per time point. The influence of bone defect size, time and interaction of these factors was assessed using the Generalised Linear Model for repeated measurements. Model effects were tested using Chi Square Wald statistics with type III analysis. Statistical comparisons between the groups per time point were performed using the MannWhitney U test (SPSS 18.0, SPSS Inc.). A $p$ value of less than 0.05 was taken as a significant difference.

\section{Results}

\section{Clinical follow-up}

Four animals from the $\mathrm{CH}$ group and one from the $\mathrm{NH}$ group were excluded from the study due to early dropouts or pin infections.

\section{In vivo setup}

At a resolution of $35.5 \mu \mathrm{m}$, repeatability tests for VOI localisation produced low variance in BV. At 6 weeks postoperatively, histological analysis did not show morphological tissue changes in the osteotomy area due to weekly and bi weekly scanning. At 6 weeks postoperative, results from $\mu \mathrm{CT}$ showed no significant differences, in callus volume fraction and tissue mineral density due to radiation between animals that received higher radiation from weekly scanning, from those that received lower radiation from bi-weekly scanning. Estimating information loss due to in vivo medium resolution scanning $(35.5 \mu \mathrm{m})$ was performed by comparing output parameters - bone volume and tissue mineral density - with high-resolution in vitro scans at $10.5 \mu \mathrm{m}$, showing no significant differences. 
(A)

(B)

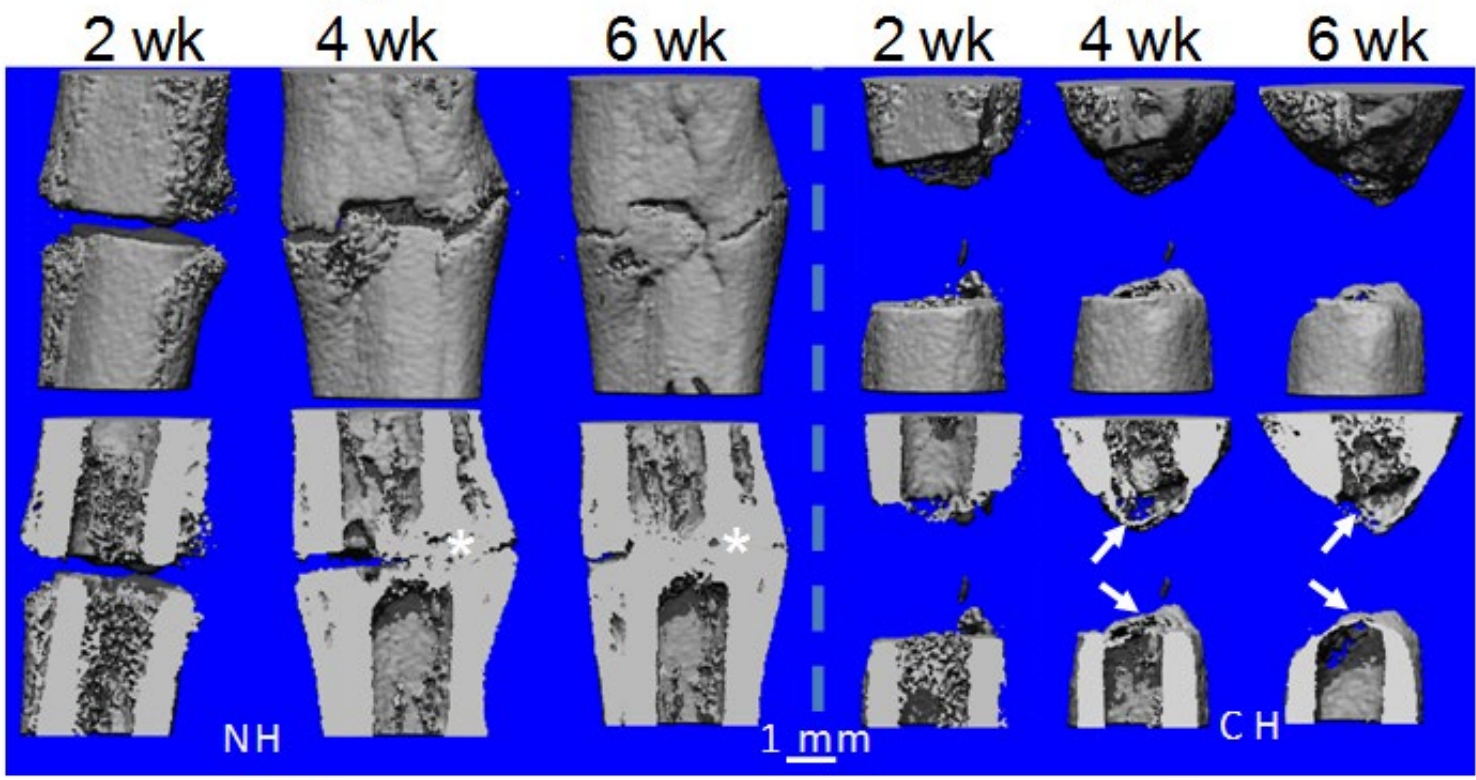

(C)

(D)
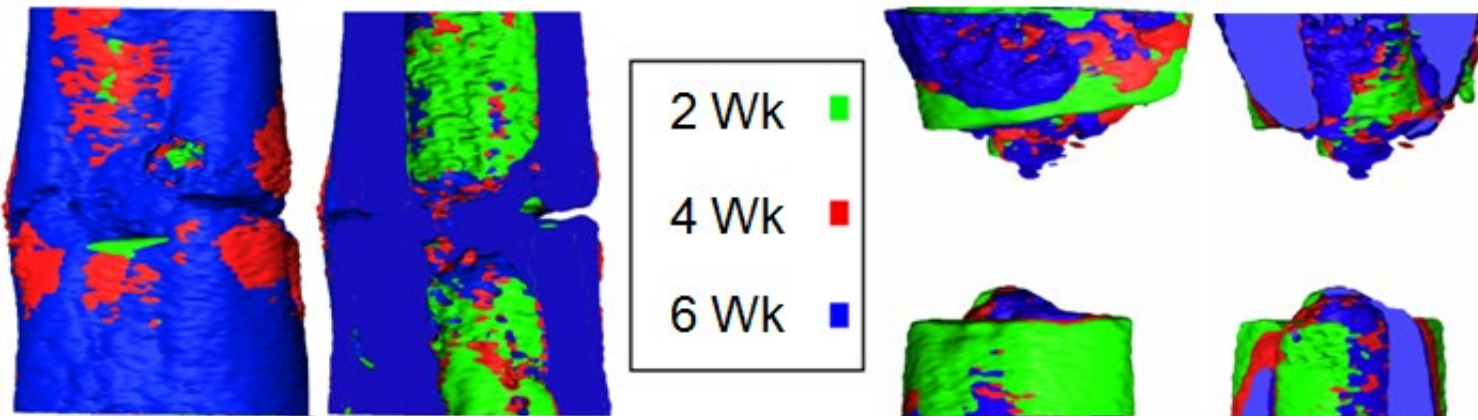

Fig. 4. Longitudinal monitoring of bone defect healing in $3 \mathrm{D}$ at 2, 4, and 6 weeks for $\mathrm{NH}$ and $\mathrm{CH}$ groups. (A) Representative images from the NH group showing callus formation in 3D at 2, 4 and 6 weeks (left of grey dashed lines). (B) Representative images from the $\mathrm{CH}$ group (right of white dashed lines). Lower row shows corresponding longitudinal cross sections of the 3D images at 2, 4 and 6 weeks, respectively, from both groups. White asterisks indicate cortical bridging in the NH group. White arrows show enclosing of the medullary cavity by means of endosteal callus formation in the $\mathrm{CH}$ group. Cortical bridging was observed at least on one side by week 4 in individuals from the $\mathrm{NH}$ group (Images in $\mathbf{A}$ ), while individuals from the $\mathrm{CH}$ group showed no such indications (images in B). Individuals from the $\mathrm{CH}$ group showed rounding of the cortex, and the development of a bony band that extended between the cortices of each fragment sealing the medullary canal from the osteotomy by week 6 (images in B). It was also observed that a slight misalignment of cortices, after fracture fixation caused the periosteal callus formation to compensate for such misalignment, shown in the NH example (images in A). During such conditions, a bony bridging occurred by means of a connection from periosteal callus to the cortex, on both sides, as seen in the longitudinal cross sections (images in A). These observations were noted as early as 2 weeks. Images $\mathbf{C}$ and $\mathbf{D}$ show $3 \mathrm{D}$ volumes of image registered data sets overlapped from scans performed at 2, 4 and 6 weeks from representative individuals in the $\mathrm{NH}(\mathbf{A})$ and $\mathrm{CH}(\mathbf{B})$ groups.

\section{Longitudinal monitoring of callus development}

Monitoring femoral bone defect healing over time demonstrated clear spatiotemporal differences in callus formation and patterning between individuals from the $\mathrm{NH}$ and $\mathrm{CH}$ groups (Figs. 4A and B). Post image registration of the data stacks from weeks 2, 4 and 6 allowed qualitative spatiotemporal visualisations of callus formation and patterning during bone healing in individuals (Figs. $4 \mathrm{C}$ and $\mathrm{D}$, Movie 1 - on paper web page). The data sets also enabled quantitative spatiotemporal characterisation of $3 \mathrm{D}$ callus development, maturation, and patterning in a region-specific manner (Figs. 5 and 6). At week 2 and onwards, periosteal callus patterning varied between the two groups (Fig. 6). The NH subjects continued to increase their callus size mainly through bone formation in periosteal regions, while $\mathrm{CH}$ subjects continued to deposit bony callus mainly in the endosteal regions (Fig. 4). Between weeks 4 and 6, the periosteal callus front and endosteal callus were observed to merge and form a cortical bony bridge in the NH group (Fig. 4). However, in the $\mathrm{CH}$ group, the periosteal callus was observed to be receding (Fig. 6B) while the endosteal callus enclosed 


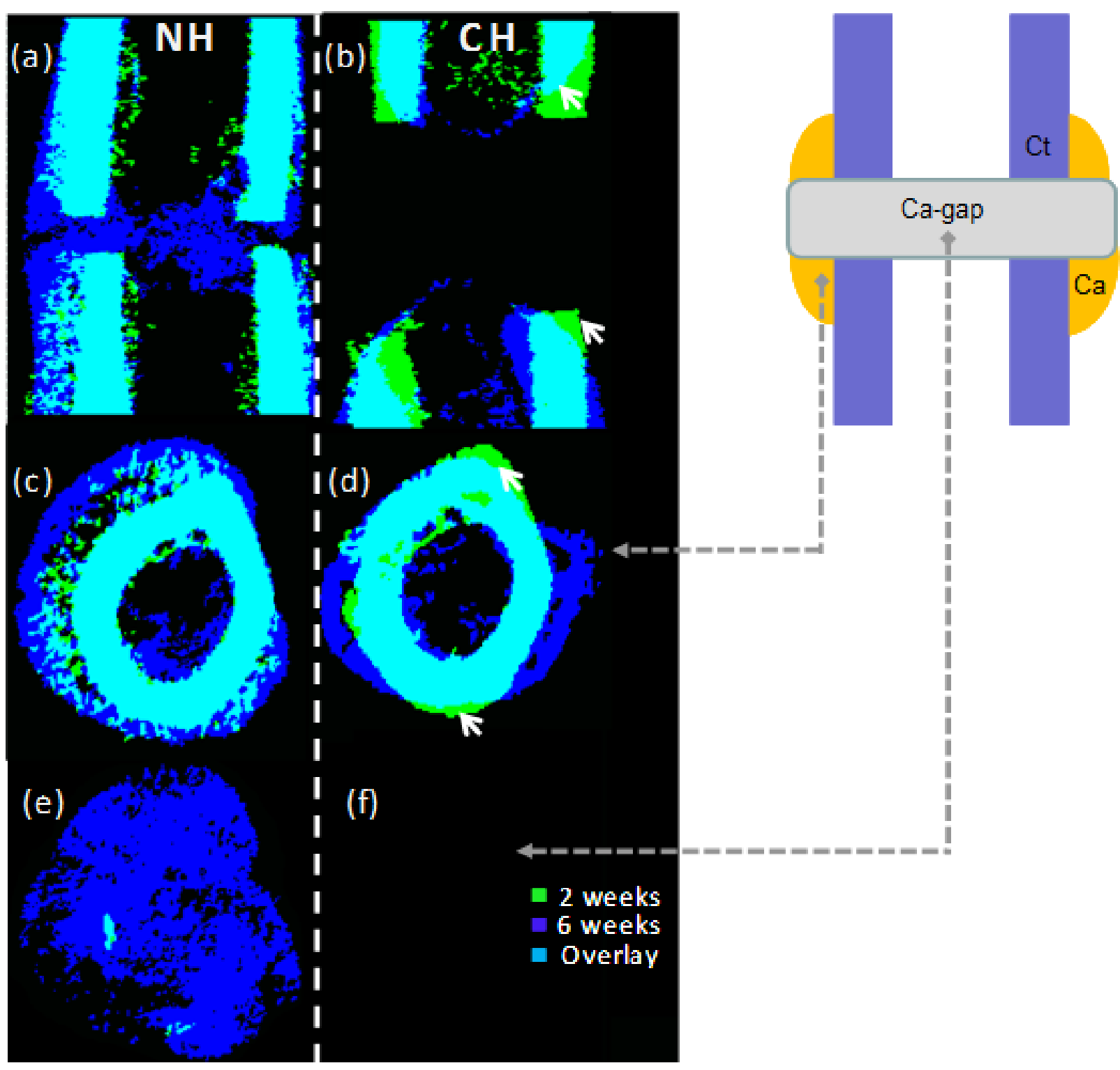

Fig. 5. Cross sections of image registered data sets overlapped from scans performed at 2, 4 and 6 weeks from representative individuals. Cross sections of images show spatiotemporal patterns of bone formation and resorption within the callus and cortex of exemplary subjects from the $\mathrm{NH}$ and $\mathrm{CH}$ groups. (A and $\mathbf{B})$ Longitudinal cross sections from the 3D volume. (C and D) Cross sections from the periosteal callus. (E and F) Images from the osteotomy gap. (F) is blank due to the lack of callus formation in the mid section of the defect region in the $\mathrm{CH}$ group. White arrows in the $\mathrm{CH}$ subject show cortical bone resorption at 6 weeks. Abbreviations are as follows: $\mathrm{Ca}$, callus; $\mathrm{Ct}$, cortical bone.

the medullary cavity (Figs. 5B and D). Unlike the NH group, no callus was observed in the midsections of the osteotomy gap in the $\mathrm{CH}$ group (Fig. 5F). Instead, bone resorption of the cortical bone ends in the $\mathrm{CH}$ subjects was noticed after week 2 that resulted in rounded cortical bone ends by week 6 (Fig. 4). The callus growth seemed to be preferential on the medial side and almost none on the lateral side of the femur. At week 6, the subjects in the $\mathrm{CH}$ group also exhibited massive cortical bone resorption, impaired periosteal callus formation, and a bony closure of the medullary canal in the osteotomy gap.

Callus formation (BV) of the femoral defect was significantly influenced by time $(p<0.001)$, bone defect size $(p<0.001)$, and their interaction $(p<0.001)$, with subjects from the NH group exhibiting a larger callus volume. It was observed that overall callus maturation, characterised by modal shifts from tissue mineral density distribution curves, was influenced by time and moved towards higher grey values over the healing period $(p<0.001)$ (Fig. 8). Callus maturation varied between the $\mathrm{NH}$ and $\mathrm{CH}$ groups $(p<0.025)$. Subjects in the $\mathrm{CH}$ group maintained a higher level of peak tissue mineralisation up to 4 weeks, in comparison with $\mathrm{NH}$ subjects (week 1: $p<0.05$, week 2: $p<0.05$, week 3: $p<0.05$, week 4: $p<0.01$, week 6: $p>0.05$ ). However, by end of 6 weeks, subjects in the $\mathrm{NH}$ group displayed higher trends in peak mineralisation. Transiently, there was a broadening of the TMDD configuration in NH groups partly showing two peaks, which indicates a strong heterogeneous distribution of the mineral in comparison with subjects in the $\mathrm{CH}$ 


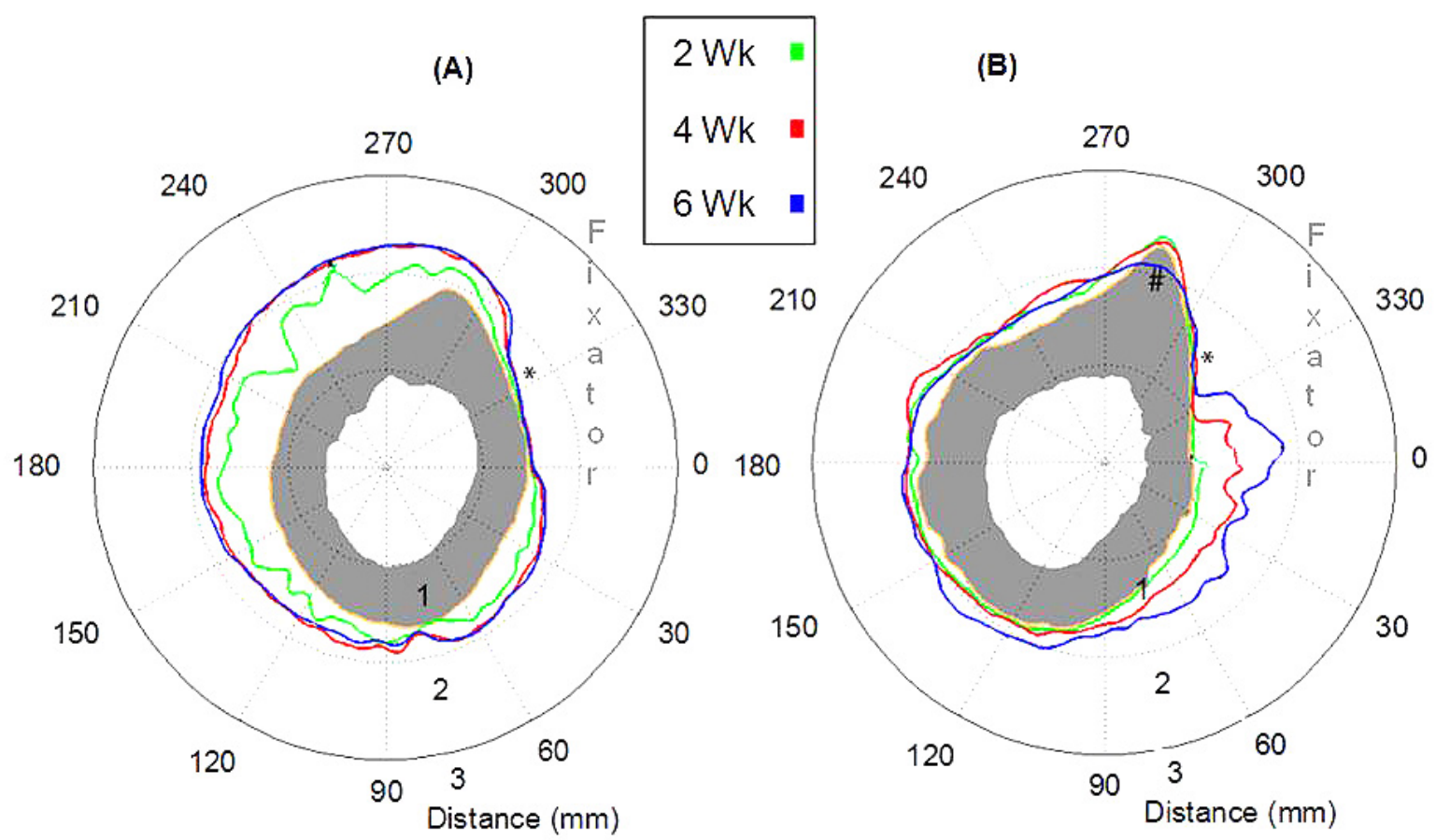

Fig. 6. Contour maps: quantitative periosteal callus patterning in polar coordinate system (degree and mm). Callus contours are extracted from image-registered data sets at weeks $0,2,4$ and 6 from representative individuals from the NH (A) and CH (B) groups. Grey areas indicate the original cortex at week 0 post operatively. (A) Individual from NH group illustrates most of periosteal callus formation taking place from week 0 to week 2. (B) Periosteal callus patterning in the $\mathrm{CH}$ group is different from the $\mathrm{NH}$ group (A). In (B), at 6 weeks the contour has moved inward at the region marked with a black hash showing callus resorption. Black asterisk show that callus formation at the fixator side (lateral) is minimal in both groups.

groups (Fig. 8). Furthermore, in the NH groups at the 6-week time point, the standard deviation is observed to be much larger than for previous time points within the group, and with comparison to the $\mathrm{CH}$ group, indicating a highly heterogeneous bone matrix with varying mineralisation regions.

\section{Region-specific bone morphometric analysis (NH $v s$. CH)}

Periosteal callus: Significant influence of healing time $(p<0.001)$, bone defect size $(p<0.001)$ and their interaction $(p<0.001)$ was observed on periosteal callus volume (BV). As early as 2 weeks, an onset of critical healing behaviour was observed in the periosteal callus due to the influence of a large bone defect size (week $2: p<0.05$, Fig. 8A). The differences between the groups remained significant over the healing period with subjects from the $\mathrm{NH}$ group producing more periosteal BV (week 3: $p<0.05$ week 4: $p<0.001$, week 6: $p<0.01$ ). Periosteal callus geometry was also observed to be influenced by time $(p<0.001)$, bone defect size $(p<0.005)$ and their interaction $(p<0.01)$. At an early onset of 2 weeks, periosteal callus geometry (pMOI) was observed to be significantly higher in $\mathrm{NH}$ subjects $(p<0.01$, Fig. $8 \mathrm{C})$, and continued to increase until week 4 . The difference in periosteal callus volume and geometry was also confirmed by contour maps that clearly indicated the spatiotemporal differences in callus patterns between the two groups (Fig. 6). BV/TV values were observed to be influenced over time $(p<0.001)$, not by defect size, but their interaction $(p<0.001)$. BV/TV differed between the two groups as early as at 2 weeks $(p<0.001$, Fig. 8 E). Subjects from the NH group showed a significant but opposite response in BV/TV at week 2 compared to $\mathrm{CH}$ subjects. They showed lesser compact callus, and then a rapid increase to higher BV/TV values by week 3 , towards a more compact callus, followed by a plateau phase, and an increase again by week $6(p<0.002)$. Mean mineralised periosteal callus tissue density (TMD) increased over healing time in all subjects $(p<0.001)$ and was observed to recover to almost $78 \pm 5.14 \%$ of intact bone (Fig. 8G). No significant difference in periosteal TMD was observed between the two groups throughout the healing period (Fig. 8G).

Osteotomy gap callus: The amount of mineralised callus volume $(\mathrm{BV})$ produced within the osteotomy gap was influenced by healing time $(p<0.001)$ and surprisingly not by the bone defect size. Interestingly, the initial response to a large size defect in $\mathrm{CH}$ group individuals was to produce more $\mathrm{BV}$ than in $\mathrm{NH}$ groups, and then losing this trend by week 3 (Fig. 8B). Upon normalising BV to the size of bone defect ( $1 \mathrm{~mm} v s .5 \mathrm{~mm})$, a significant influence of relative callus formation rate was observed between the two groups ( $p<0.001$, Fig. 8B). As early as 2 weeks, an onset of critical healing patterns was observed in the osteotomy gap callus due to the influence of large bone defect size (week 2: $p<0.001$, Fig. 8B). The differences between the groups remained significant over the healing period, with subjects from the NH group producing higher 
Fig. 7. TMDD curves of all subjects for the three time points show overall callus maturation (mineralisation kinetics and heterogeneity of material) in $\mathrm{NH}$ and $\mathrm{CH}$ groups. Shaded areas show the variation from the average TMDD curve. Circles refer to peak and squares refer to the half maximum (Fig. 3). In both groups there was a shift of the curves towards higher mineralisation over time, shown by black arrows. The peak positions of the curves moved right towards higher mineral levels, the width of curves at half full width also got larger, shown by the black dashed arrow.
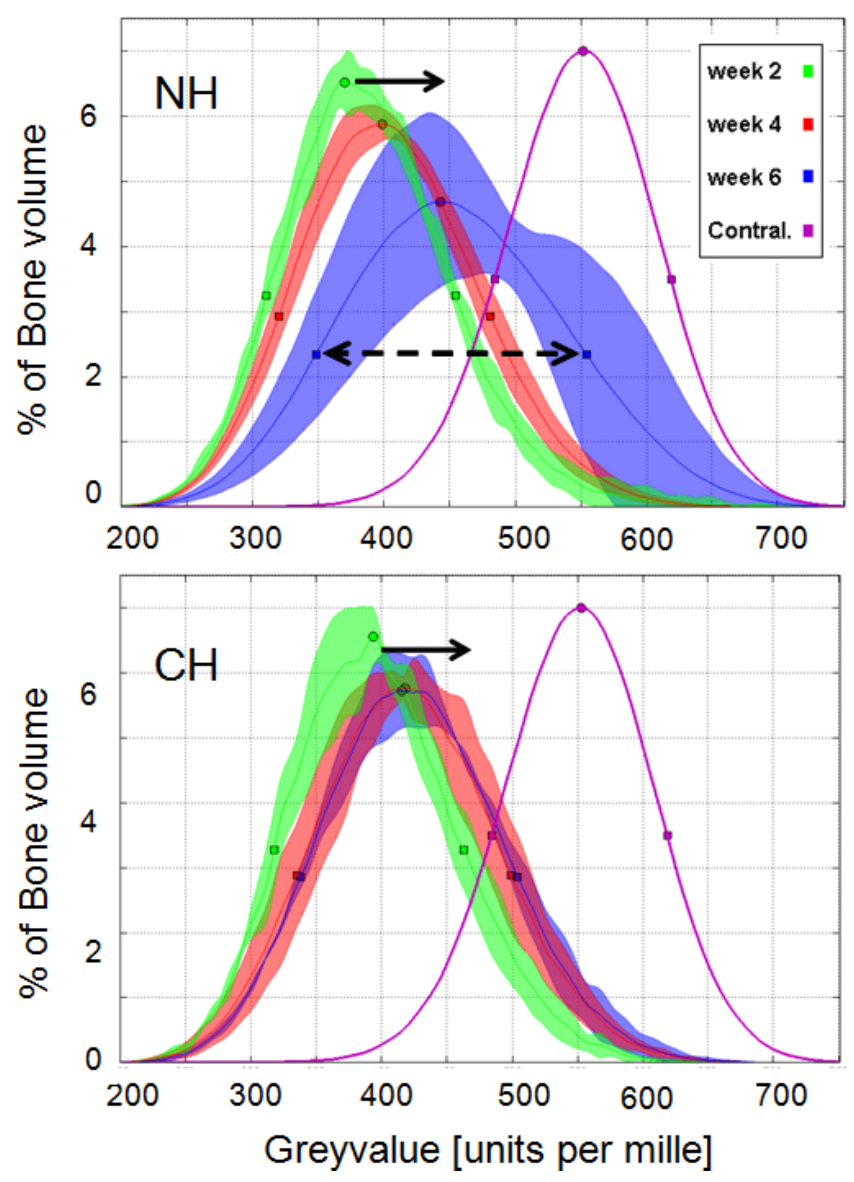

relative $\mathrm{BV}$ in the osteotomy gap (week $3: p<0.05$, week 4: $p<0.01$, week 6: $p<0.01$ ). Callus geometry (pMOI) in the osteotomy gap was observed to be influenced by time $(p<0.001)$, bone defect size $(p<0.001)$ and their interaction $(p<0.001)$. At an early onset of 2 weeks, osteotomy callus geometry (pMOI) was observed to be significantly higher in NH subjects ( $p<0.05$, Fig. 8D) and continued to increase over time. In contrast, the bony callus in the $\mathrm{CH}$ group individuals was closely distributed around the central axis, enclosing the medullary cavity, and therefore resulting in very low pMOI values in comparison with the NH group (Figs. 8D and 4). BV/TV values were observed to be influenced over time $(p<0.001)$, defect size $(p<0.05)$, and their interaction $(p<0.001)$. Subjects from the $\mathrm{CH}$ group showed a delayed response until week 4 , but increased to higher values by week $6(p<0.05$, Fig. $8 \mathrm{~F}$ ). Mean mineralised osteotomy callus tissue density (TMD \%) increased over healing time in all subjects ( $p<0.001$ ), with $\mathrm{CH}$ individuals displaying higher TMD at week 4. No significant difference in TMD was observed due to bone defect size at week 6 (Fig. $8 \mathrm{H})$, and was observed to recover to almost $73.6 \pm 12.7 \%$ of intact bone. It was observed that early callus at 1 week in all subjects had very high mineral density followed by a reduction in mineral density at week 2 , and then again an onward increasing mineralisation until $74 \%$. A full recovery to intact bone tissue mineralisation was not observed in the primary phase.

Week 8 in $\mathrm{CH}$ individuals showed no signs of a delayed healing, rather, it confirmed the bony non-union, reduced periosteal callus size and geometry (pMOI), massive cortical resorption, and highly organised bone structure (TMD) in the osteotomy callus (Fig. 8).

\section{Strain patterns in the callus region}

A marked difference in strain patterns was predicted in the normal and critical healing situations (Fig. 9). Especially interesting were the differences in strain patterns observed in the endosteal gap region. While significant levels of strain were predicted deep in the endosteal region in the $\mathrm{NH}$ group, coincident with bone formation patterns at later time points, in the $\mathrm{CH}$ group the same levels of strain were only reached deep into the osteotomy gap region where new bone formation closing up the marrow cavity was observed at later stages (Fig. 4D). In both groups the highest levels of strain were predicted in the intra-cortical region moving out radially into the periosteal areas (Fig. 9), however the $\mathrm{CH}$ group exhibited significantly lower levels of strain compared to the NH group.

\section{Discussion}

To the authors' knowledge, the novel in vivo tracking method developed in this study that allows for 3D spatiotemporal monitoring of a callus in a region-specific manner, allowing for image registration possibilities, while enabling quantitative characterisation of callus volume, geometry, patterning and mineralisation kinetics - with region specificity - over the time course of healing has not 


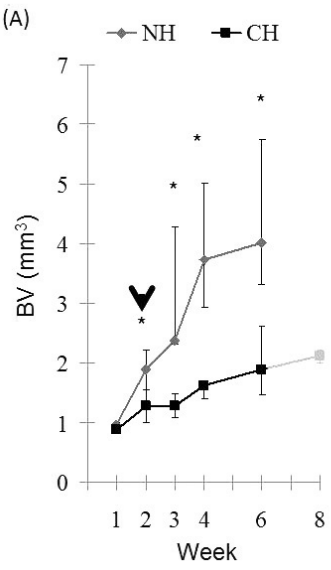

(E)

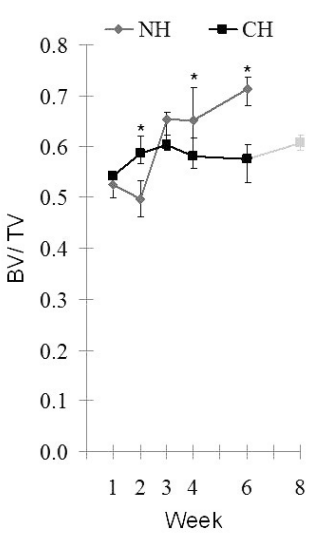

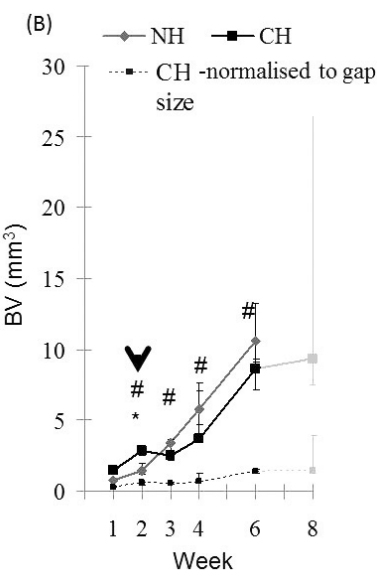

(F)

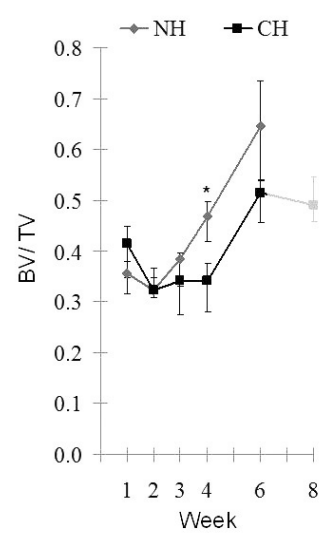

(C)

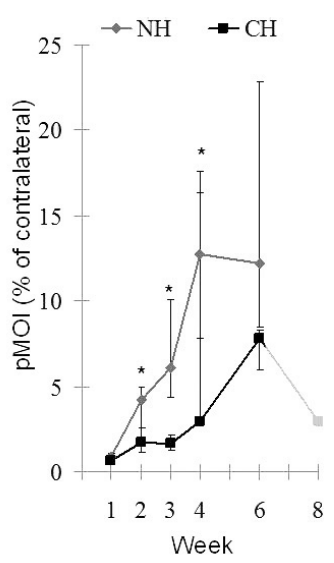

(G)

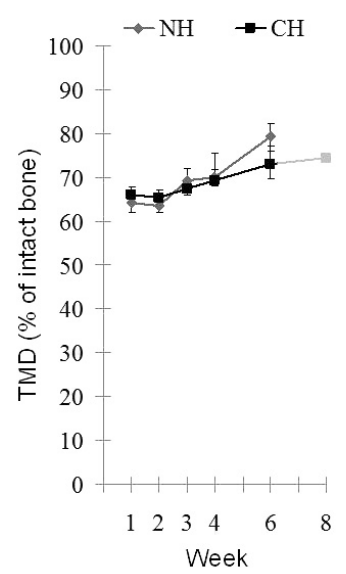

(D)

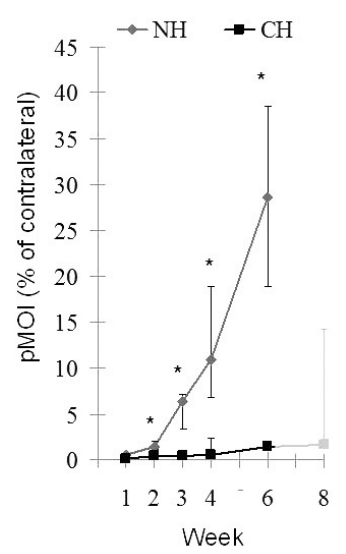

(H)

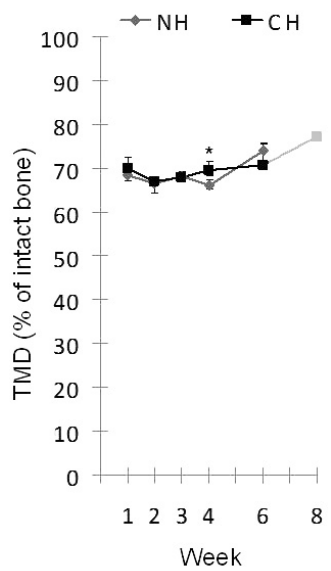

Fig. 8. 4D bone morphometric analysis for comparison between $\mathrm{NH}$ and $\mathrm{CH}$ groups. Images show the region specific analysis ( $\mathbf{A}, \mathbf{C}, \mathbf{E}$ and $\mathbf{G}$ - periosteal callus region; $\mathbf{B}, \mathbf{D}, \mathbf{F}$ and $\mathbf{H}$ - osteotomy gap callus region). Image $\mathbf{B}$ contains plots for BV (NH and CH groups), and overlay plot for normalised BV to fracture gap (normalised data shown only for $\mathrm{CH}$ group, $\mathrm{Y}$ scale not adjusted for normalised data). Black arrows in images indicate onset of critical healing patterns in the $\mathrm{CH}$ group in comparison with the $\mathrm{NH}$ group. The grey regions indicate the additional two weeks (total: 8 weeks) of monitoring in the $\mathrm{CH}$ groups.

been previously shown. Previous studies using $\mu \mathrm{CT}$ as a tool to characterise healing outcome have not been able to track in vivo 3D changes of callus features in a regionspecific manner. The study results have demonstrated that callus volume, geometry and patterning, but not callus mineralisation, are impaired during large bone defect healing that lead to atrophic non-unions. Furthermore, by implementing the experimental data into FE models, the study results have indicated that predicted mechanical strains in the early reparative phase are similar to the patterns of callus formation during bone defect healing. Using region-specific in-depth quantitative characterisations, the study clearly demonstrates that: (i) periosteal bone formation and patterning are significantly influenced by bone defect size as early as 2 weeks; (ii) osteotomy gap callus formation and patterning are significantly influenced by large sized bone defects, and take a deviation into the medullary route as early as 2 weeks; (iii) the new bone formation in the osteotomy gap enclosing the medullary cavity in the $\mathrm{CH}$ group is highly mineralised, and patterned in a different manner in comparison with $\mathrm{NH}$ groups; (iv) inter-fragmentary strain patterns predicted during the very early soft callus tissue phase are concurrent with callus formation and patterning at later stages.

Using the in vivo setup, it was possible to select the volume of interest repeatedly in the scanning volume, making it easier to run standard image registration rigid body correlation techniques. This novel feature also made it possible to run bone morphometric analysis and quantify callus patterning, formation and heterogeneity of mineralisation of the callus over time of a constant VOI with region-specific resolution. It has been shown that no influence of radiation was observed when scanning was increased from bi-weekly to weekly. The calculated dosage index of $86 \mathrm{mGy}$ in this study is lower than the amounts that have been reported and confirmed safe to use. Furthermore, the in vivo monitoring system using $35.5 \mu \mathrm{m}$ resolution provided sufficient quality when compared with high-resolution in vitro scans.

In a previous study, subjects with a $1 \mathrm{~mm}$ bone gap size achieved bony bridging and a biomechanical stiffness up to $75 \%$ of the intact contralateral limb by week 6 
$\mathrm{NH}$ group

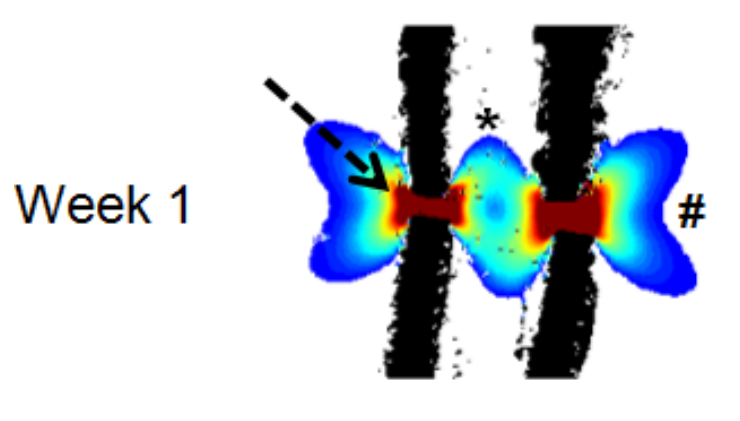

Week 2
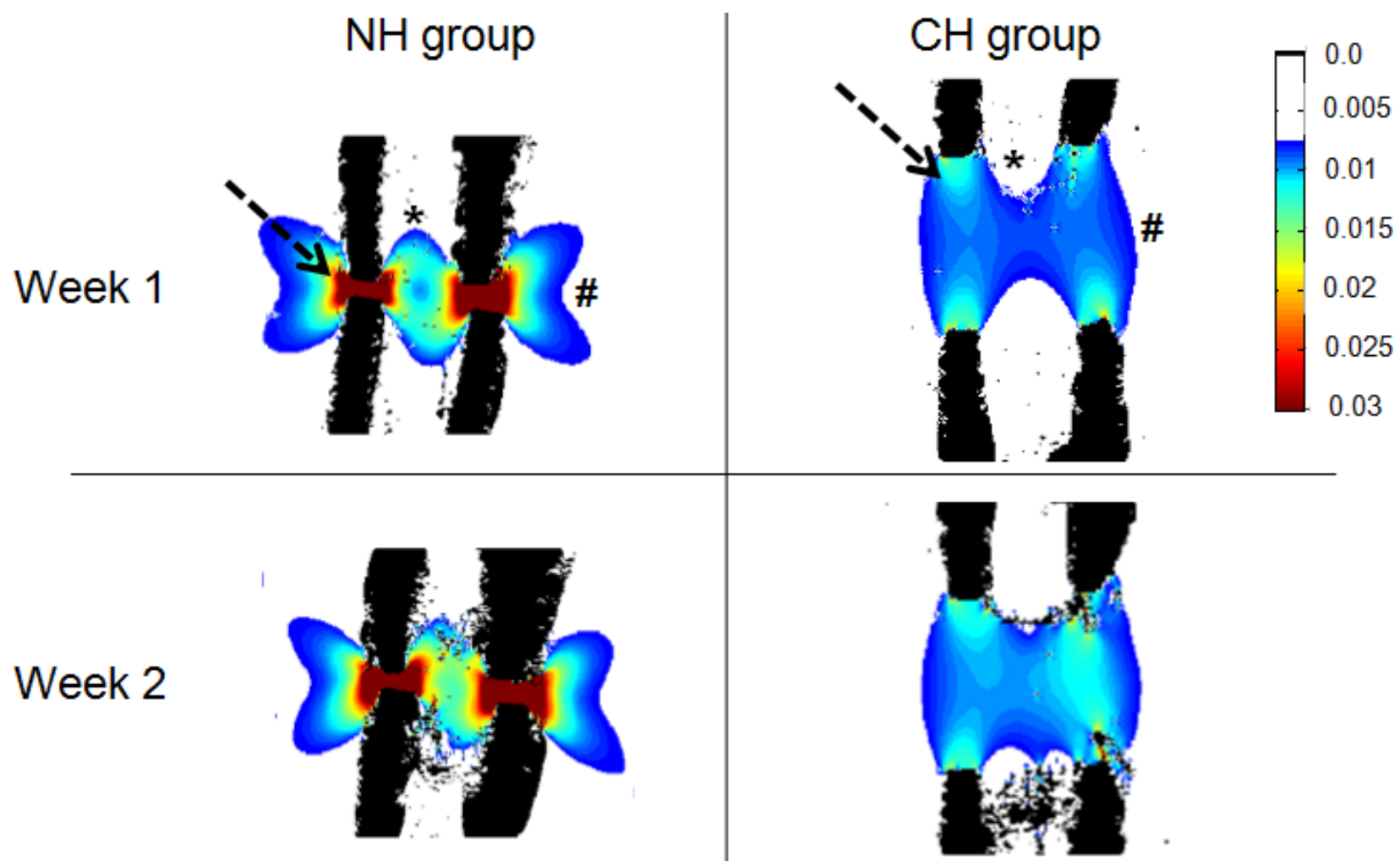

Fig. 9. Differing minimum principal strain patterns in the callus region of $\mathrm{NH}$ and $\mathrm{CH}$ groups during early phase healing. Differences between the two groups are emphasised by an arrow, asterisk and hash. In the normal healing model, the highest levels of strain were predicted in the intra-cortical region moving out radially into the periosteal areas (shown by dashed arrow). In the critical healing model, strain levels in the whole callus region were lower and occurred deep in the osteotomy gap where new bone formation was observed during later stages of healing (shown by asterisk).

(Mehta et al., 2011b). Therefore, we used subjects with a $1 \mathrm{~mm}$ bone defect as a control group showing normal healing patterns $(\mathrm{NH})$, and compared it to critical healing patterns in a $5 \mathrm{~mm}$ segmental bone $\operatorname{defect}(\mathrm{CH})$ that resulted in atrophic non-unions. Bone volume fraction, callus patterning, and geometry of callus were influenced due to a segmental defect as early as 2 weeks. The study has also shown that callus formation is clearly not symmetric along the longitudinal axis of the femur and must be accounted for during 2D sectioning during histological studies (Gerstenfeld et al., 2006; Lujan et al., 2010), and investigated further using computer simulations in mechanobiology of bone regeneration. The medial side of femurs showed the largest callus formation indicating the likely influence of the surrounding bulky and nontraumatised soft muscle tissue because of the lateral surgical approach in this animal model.

This in-depth study has furthered our understanding of callus formation and patterning during endogenous bone defect healing. Particularly, it has been shown that periosteal callus is an early responder and strongly influenced by the size of a bone defect. The function of the periosteal callus area may be to quickly stabilise the defect area by mineralised callus volume (BV) that contributes directly towards increasing the polar moment of inertia (PMOI) of the mineralised callus (Mehta et al., 2010). The need for stability seems to come as a response to straining of early tissue within the bone defect environment. On establishing the required stability by increasing PMOI, the periosteal callus then moves into a plateau phase, wherein the callus matures by becoming more compact (increasing $\mathrm{BV} / \mathrm{TV}$ ), and increasing mineralisation (TMD). The osteotomy callus was observed to exhibit regular healing patterns in the normal healing groups. However, in the critical healing group, due to the large bone defect size, the osteotomy gap callus exhibited more intramembranous formation behaviour.

Callus mechanical competence depends on a variety of parameters such as volume, geometry (pattern), tissue mineral density (TMD), and extent of mineralisation (TMDD). Mineral content of the callus is known to strongly influence bone strength, and thereby the spatial and temporal variations of mineral content caused by callus formation and mineralisation will lead to variations in the mechanical properties of bone material. Use of $\mu \mathrm{CT}$ has been shown to permit such tissue-level characterisation of average and density distribution of mineral using quantitative methods (Burghardt et al., 2008; Kazakia et al., 2008; Mulder et al., 2004). The heterogeneous distribution of mineral content in trabecular bone reflects the continuous renewal of bone material in bone remodelling and the subsequent increase in mineral content in the newly formed bone packets. Using the longitudinal monitoring setup, this study has enabled a 4D measurement tool that quantifies the dynamics of mineralisation of callus tissue during bone healing in individuals. Following the 
TMDD peaks over time, this study suggests that individuals in the $\mathrm{NH}$ group roughly have a mineralisation rate of $7 \%$ per 2 weeks towards regaining of bone strength. It has been shown that normal healing produces a more heterogeneous tissue, indicated by the increasing TMDD full width over time. In contrast, the $\mathrm{CH}$ group showed a consistent narrow TMDD full width indicating a more homogenous tissue development. These results support the tissue patterns we have observed in histological sections from our previous study (Mehta et al., 2011b), wherein callus produced in $\mathrm{NH}$ groups are of woven bone structure and those in $\mathrm{CH}$ groups are of a more lamellar bone type. Furthermore, a full recovery to $100 \%$ bone tissue mineralisation was not observed in the 6-8 week follow-up phase of healing. Our previous studies have also shown similar results that callus mineralisation occurs only to $75-80 \%$ during the bony bridging phase.

Although, the accuracy of $\mu \mathrm{CT}$ with polychromatic radiation can be considered adequate for assessment of the degree of mineralisation of developing bone (Burghardt et al., 2008), this study does not report absolute numbers on degree of mineralisation. Instead, bone mineral density distribution has been used as a tool to compare relative kinetics ( $\%$ of intact bone) within groups. More accurate measurement methods in bone mineral density distribution are available, such as quantitative backscattered electron imaging (qBEI) (Roschger et al., 1998), or 3D synchrotron (Burghardt et al., 2008). The drawbacks with qBEI or synchrotron methods are that: (i) the determination of BMDD requires undecalcified bone samples; (ii) equipment is not easily accessible; (iii) these require in vitro non living samples.

Using experimental data of the early phases of bone healing in combination with finite element analysis, the influence of a large defect size on strain field patterns in an osteotomy gap, and consequently its relationship to the ossification pattern during healing was investigated. A constant displacement will result in an increase of the force applied to the models as they gradually become stiffer. However, as can be seen in Fig. 9, very small amounts of bone formation occurred between weeks 1 and 2 which may not alter the strain distributions within the gap. Furthermore, in our previous study (Mehta et al., 2011b), we have also observed that no cartilage formation has taken place that may influence the stiffness within the gap. Strain fields predicted in the $\mathrm{NH}$ and $\mathrm{CH}$ group significantly differed in their patterns in the periosteal and osteotomy regions. Strain levels below $3 \%$ have been proposed as favourable for bone formation (Claes and Heigele, 1999; Prendergast et al., 1997), which are in accordance with our model predictions. Callus patterning was observed to follow the footprint of tissue strain patterns, especially in the endosteal region. In the NH group, strains over $0.7 \%$ were predicted deep into the medullary canal, where bone formation was experimentally observed. In the $\mathrm{CH}$ group, these ranges of strain only occurred deep into the gap region with a patterning that resembled the closure of the medullary canal at later time points. Furthermore, within the osteotomy gap, strain fields in the $\mathrm{CH}$ group were lower than in $\mathrm{NH}$ groups possibly influencing the callus formation rate and patterning.

\section{Conclusion}

It has been shown that callus formation, geometry and patterning, but not tissue mineralisation, are strongly affected by early mechanical stimulus during bone defect healing. A powerful method of qualitative and quantitative 4D (3D + longitudinal dimension) monitoring of callus structure, patterning and mineralisation in a region-specific manner has been described in this study. Such a detailed follow-up using 4D monitoring in bone defect healing can be valuable in spatiotemporal tissue engineering strategies using scaffolds to promote musculoskeletal tissue regeneration (Mehta et al., 2012).

Contrary to previous tissue engineering approaches using scaffolds of very high stiffness in bone defects, an alternative strategy would be tuneable or softer scaffold stiffness that guide callus formation and patterns. Recent studies have demonstrated that progenitor cells are responsive to local scaffold stiffness (Discher et al., 2009). Consequently, it has been shown that engineering scaffold stiffness can influence osteogenic behaviour of progenitor cells (DeForest and Anseth, 2012; Huebsch et al., 2010). Based on our current study results indicating that bone forms in regions where strain levels are around $1 \%$ during the early phase of healing, a promising approach would be to engineer scaffolds for large sized bone defects with a stiffness that would lead to strain patterns similar to those observed in the $\mathrm{NH}$ groups during the very early soft callus tissue phase. The tools developed in this study can be applied in characterising and designing such new approaches.

\section{Acknowledgments}

This research was supported in part by grants from the Berlin-Brandenburg Centre for Regenerative Therapies (BCRT), and by a grant of the German Research Foundation (SFB 760), and Nachwuchsakademie Medizintechnik (DFG- ME 4083/1-1). The authors would like to thank Mr. Mark Heyland, Miss Anna Woloszyk and Mr. Mario Thiele for their excellent technical assistance. The authors would like to thank Dr. Agnes Ellinghaus and Dr. Katharina Schmidt-Bleek for assisting with animal surgeries. We wish to confirm that there are no known conflicts of interest associated with this publication and there has been no significant financial support for this work that could have influenced its outcome.

\section{References}

Burghardt A, Kazakia G, Laib A, Majumdar S (2008) Quantitative assessment of bone tissue mineralization with polychromatic micro-computed tomography. Calcif Tissue Int 83: 129-138.

Checa S, Prendergast PJ, Duda GN (2011) Inter-species investigation of the mechano-regulation of bone healing: comparison of secondary bone healing in sheep and rat. $\mathrm{J}$ Biomech 44: 1237-1245. 
Claes LE, Heigele CA (1999) Magnitudes of local stress and strain along bony surfaces predict the course and type of fracture healing. J Biomech 32: 255-266.

Claes L, Augat P, Suger G, Wilke HJ (1997) Influence of size and stability of the osteotomy gap on the success of fracture healing. Journal of Orthopaedic Research 15: 577-584.

Claes LE, Heigele CA, Neidlinger-Wilke C, Kaspar D, Seidl W, Margevicius KJ, Augat P (1998) Effects of mechanical factors on the fracture healing process. Clin Orthop Relat Res 355 Suppl: S132-147.

DeForest CA, Anseth KS (2012) Advances in bioactive hydrogels to probe and direct cell fate. Annu Rev Chem Biomol Eng 3: 421-444.

Discher DE, Mooney DJ, Zandstra PW (2009) Growth factors, matrices, and forces combine and control stem cells. Science 324: 1673-1677.

Duda GN, Eckert-Hubner K, Sokiranski R, Kreutner A, Miller R, Claes L (1998) Analysis of inter-fragmentary movement as a function of musculoskeletal loading conditions in sheep. J Biomech 31: 201-210.

Duvall CL, Taylor WR, Weiss D, Wojtowicz AM, Guldberg RE (2007) Impaired angiogenesis, early callus formation, and late stage remodeling in fracture healing of osteopontin-deficient mice. J Bone Miner Res 22: 286-297.

Gerstenfeld LC, Alkhiary YM, Krall EA, Nicholls FH, Stapleton SN, Fitch JL, Bauer M, Kayal R, Graves DT, Jepsen KJ, Einhorn TA (2006) Three-dimensional reconstruction of fracture callus morphogenesis. J Histochem Cytochem 54: 1215-1228.

Goodship AE, Kenwright J (1985) The influence of induced micromovement upon the healing of experimental tibial fractures. J Bone Joint Surg Br 67: 650-655.

Hildebrand T, Ruegsegger P (1997) A new method for the model-independent assessment of thickness in threedimensional images. J Microsc 185: 67-75.

Huebsch N, Arany PR, Mao AS, Shvartsman D, Ali OA, Bencherif SA, Rivera-Feliciano J, Mooney DJ (2010) Harnessing traction-mediated manipulation of the cell/ matrix interface to control stem-cell fate. Nat Mater 9: 518-526.

Kazakia GJ, Burghardt AJ, Cheung S, Majumdar S (2008) Assessment of bone tissue mineralization by conventional $\mathrm{x}$-ray microcomputed tomography: comparison with synchrotron radiation microcomputed tomography and ash measurements. Med Phys 35: 3170 3179 .

Kenwright J, Goodship AE (1989) Controlled mechanical stimulation in the treatment of tibial fractures. Clin Orthop Relat Res 241: 36-47.

Kraus KH, Kadiyala S, Wotton H, Kurth A, Shea M, Hannan M, Hayes WC, Kirker-Head CA, Bruder S (1999) Critically sized osteo-periosteal femoral defects: a dog model. J Invest Surg 12: 115-124.

Lacroix D, Prendergast PJ (2002) A mechanoregulation model for tissue differentiation during fracture healing: analysis of gap size and loading. J Biomech 35: 1163-1171.
Leong PL, Morgan EF (2008) Measurement of fracture callus material properties via nanoindentation. Acta Biomater 4: 1569-1575.

Lujan TJ, Henderson CE, Madey SM, Fitzpatrick DC, Marsh JL, Bottlang M (2010) Locked plating of distal femur fractures leads to inconsistent and asymmetric callus formation. J Orthop Trauma 24: 156-162.

Marsh D (1998) Concepts of fracture union, delayed union, and nonunion. Clin Orthop Relat Res 355 Suppl: S22-30.

Mehta M, Strube P, Peters A, Perka C, Hutmacher D, Fratzl P, Duda GN (2010) Influences of age and mechanical stability on volume, microstructure, and mineralization of the fracture callus during bone healing: is osteoclast activity the key to age-related impaired healing? Bone 47: 219-228.

Mehta M, Duda GN, Perka C, Strube P (2011a) Influence of gender and fixation stability on bone defect healing in middle-aged rats: A pilot study. Clin Orthop Relat Res 469: 3102-3110.

Mehta M, Schell H, Schwarz C, Peters A, SchmidtBleek K, Ellinghaus A, Bail HJ, Duda GN, Lienau J (2011b) A 5-mm femoral defect in female but not in male rats leads to a reproducible atrophic non-union. Arch Orthop Trauma Surg 131: 121-129.

Mehta M, Schmidt-Bleek K, Duda GN, Mooney DJ (2012) Biomaterial delivery of morphogens to mimic the natural healing cascade in bone. Adv Drug Deliv Rev 64: 1257-1276.

Morgan EF, Mason ZD, Chien KB, Pfeiffer AJ, Barnes GL, Einhorn TA, Gerstenfeld LC (2009) Micro-computed tomography assessment of fracture healing: Relationships among callus structure, composition, and mechanical function. Bone 44: 335-344.

Mulder L, Koolstra JH, Van Eijden TM (2004) Accuracy of microCT in the quantitative determination of the degree and distribution of mineralization in developing bone. Acta Radiol 45: 769-777.

Prendergast PJ, Huiskes R, Soballe K (1997) Biophysical stimuli on cells during tissue differentiation at implant interfaces. J Biomech 30: 539-548.

Reichert JC, Cipitria A, Epari DR, Saifzadeh S, Krishnakanth P, Berner A, Woodruff MA, Schell H, Mehta M, Schuetz MA, Duda GN, Hutmacher DW (2012) A tissue engineering solution for segmental defect regeneration in load-bearing long bones. Sci Transl Med 4: 141ra93.

Roschger P, Fratzl P, Eschberger J, Klaushofer K (1998) Validation of quantitative backscattered electron imaging for the measurement of mineral density distribution in human bone biopsies. Bone 23: 319-326.

Ruffoni D, Fratzl P, Roschger P, Klaushofer K, Weinkamer R (2007) The bone mineralization density distribution as a fingerprint of the mineralization process. Bone 40: 1308-1319.

Shefelbine SJ, Simon U, Claes L, Gold A, Gabet Y, Bab I, Muller R, Augat P (2005) Prediction of fracture callus mechanical properties using micro-CT images and voxel-based finite element analysis. Bone 36: 480-488. 
Strube P, Mehta M, Putzier M, Matziolis G, Perka C, Duda GN (2008) A new device to control mechanical environment in bone defect healing in rats. J Biomech 41: 2696-2702.

van Lenthe GH, Muller R (2008) CT-based visualization and quantification of bone microstructure in vivo. IBMS BoneKEy 5: 410-425.

Wehner T, Wolfram U, Henzler T, Niemeyer F, Claes L, Simon U (2010) Internal forces and moments in the femur of the rat during gait. J Biomech 43: 2473-2479.

\section{Discussion with Reviewers}

Reviewer II: Applying unchanging displacements to the FE models obtained from animals at different stages of healing will give an increasing force applied as the models gradually stiffen with healing. This approach needs to be justified against simply applying physiological loads to the FE models. Why were the displacements applied to the $\mathrm{CH}$ animals smaller?

Authors: We completely agree with the reviewer and apologise for the lack of clarity. A constant displacement will result in an increase of the force applied to the models as they gradually become stiffer. However, as can be seen in Fig. 9, very small amounts of bone formation occurred between weeks 1 and 2 which will not considerably alter the strain distributions within the gap. Furthermore, in our previous study (Mehta et al., 2011b), we did not observed cartilage formation that may influence the stiffness within the gap.

The displacements were calculated from a full 3D model that included the external fixator, where a compression load was applied externally to the bone and the IFM at the gap determined. The different gap sizes resulted in slightly different IFMs which where thereafter applied to the CT-scan-derived 2D models.

Reviewer II: The obtained result on strain magnitudes is not surprising given the large difference in length of the $\mathrm{CH}$ and $\mathrm{NH}$ callus and the similar size of the displacements applied. What happens if load control rather than displacement control is used?

Authors: Since our $\mu$ CT-based two dimensional models do not include the external fixator, the application of a physiological load to these models would not be realistic. To determine the amount of load transferred to the gap when the external fixator is mounted, we have made use of a previously developed 3D model that includes the external fixator (Checa et al., 2011). In this way, we were able to determine how changes in the gap size affect how much load is transmitted to the gap. From these 3D models, we determined the IFM for each of the gap sizes that we used as our loading condition. It would have been equivalent to determine the amount of load that is transmitted to the gap for each gap size. However, those forces would have been different for the critical and uneventful healing cases.

It would be equivalent to perform a load-controlled simulation as far as the loads applied to each model, uneventful and critical size, correspond to the loads that are transferred to the gap region when the external fixator is mounted. These loads are different for different gap sizes under the same fixation system. In this study, we have determined the differences in the IFM under the same fixation system for different gap sizes and use those values as loading conditions. In the same way, it would be possible to determine the specific boundary conditions for each model as loads, which would provide the same results. 